\title{
BMJ Open Detection of subclinical rheumatic heart disease in children using a deep learning algorithm on digital stethoscope: a study protocol
}

\author{
Fatima Ali (1) , ${ }^{1}$ Babar Hasan, ${ }^{1}$ Huzaifa Ahmad, ${ }^{2}$ Zahra Hoodbhoy (D) , ${ }^{1}$ \\ Zainab Bhuriwala, ${ }^{1}$ Muhammad Hanif, ${ }^{3}$ Shahab U Ansari, ${ }^{3}$ Devyani Chowdhury ${ }^{4}$
}

To cite: Ali F, Hasan B, Ahmad $\mathrm{H}$, et al. Detection of subclinical rheumatic heart disease in children using a deep learning algorithm on digital stethoscope: a study protocol. BMJ Open 2021;11:e044070. doi:10.1136/ bmjopen-2020-044070

- Prepublication history and additional supplemental material for this paper are available online. To view these files, please visit the journal online (http://dx.doi.org/10.1136/ bmjopen-2020-044070).

FA and $\mathrm{BH}$ are joint first authors.

Received 21 August 2020 Accepted 28 July 2021

\section{Check for updates}

(c) Author(s) (or their employer(s)) 2021. Re-use permitted under CC BY-NC. No commercial re-use. See rights and permissions. Published by BMJ.

${ }^{1}$ Pediatrics and Child Health, Aga Khan University Hospital, Karachi, Pakistan

${ }^{2}$ Medicine, MedStar Georgetown University Hospital, Washington, District of Columbia, USA

${ }^{3}$ Faculty of Computer Science and Engineering, Ghulam Ishac Khan Institute of Engineering Sciences and Technology, Topi, Pakistan

${ }^{4}$ Cardiology, Cardiology care for Children, Lancaster PA, Al DuPont Children's Hospital, Wilmington, Delaware, USA

Correspondence to Dr Devyani Chowdhury; dchowdhury@ cardiologylancaster.com

\section{ABSTRACT}

Introduction Rheumatic heart diseases (RHDs) contribute significant morbidity and mortality globally. To reduce the burden of RHD, timely initiation of secondary prophylaxis is important. The objectives of this study are to determine the frequency of subclinical RHD and to train a deep learning (DL) algorithm using waveform data from the digital auscultatory stethoscope (DAS) in predicting subclinical RHD.

Methods and analysis We aim to recruit 1700 children from a group of schools serving the underprivileged over a 12-month period in Karachi (Pakistan). All consenting students within the age of 5-15 years with no underlying congenital heart disease will be eligible for the study. We will gather information regarding sociodemographics, anthropometric data, history of symptoms or diagnosis of rheumatic fever, phonocardiogram (PCG) and electrocardiography (ECG) data obtained from DAS. Handheld echocardiogram will be performed on each study participant to assess the presence of a mitral regurgitation (MR) jet $(\geq 1.5 \mathrm{~cm})$, or the presence of aortic regurgitation $(A R)$ in any view. If any of these findings are present, a confirmatory standard echocardiogram using the World Heart Federation (WHF) will be performed to confirm the diagnosis of subclinical RHD. The auscultatory data from digital stethoscope will be used to train the deep neural network for the automatic identification of patients with subclinical RHD. The proposed neural network will be trained in a supervised manner using labels from standard echocardiogram of the participants. Once trained, the neural network will be able to automatically classify the DAS data in one of the three major categories-patient with definite RHD, patient with borderline RHD and normal subject. The significance of the results will be confirmed by standard statistical methods for hypothesis testing.

Ethics and dissemination Ethics approval has been taken from the Aga Khan University, Pakistan. Findings will be disseminated through scientific publications and to collaborators.

Article focus This study focuses on determining the frequency of subclinical RHD in school-going children in Karachi, Pakistan and developing a DL algorithm to screen for this condition using a digital stethoscope.
Strengths and limitations of this study

- This study will be the first in Pakistan to develop a deep learning algorithm on phonocardiogram and electrocardiogram waveform data from the digital stethoscope to screen for subclinical rheumatic heart disease.

- To detect the frequency of subclinical rheumatic heart disease, all study participants will be screened using handheld echocardiograms and those with positive findings will have a confirmatory standard echocardiogram to ensure accurate diagnosis.

- Study participants will be selected from one group of schools. However, the demographics of this school group are generalisable to the larger population of Pakistani school-going children.

\section{INTRODUCTION}

Rheumatic heart disease (RHD) is a serious complication of childhood acute rheumatic fever, that develops following an abnormal immune response to a beta-haemolytic streptococcal pharyngitis infection. ${ }^{1}$ If left untreated, patients are at risk of established valvular lesions and eventual heart failure. ${ }^{2}$ Globally, RHD contributes to significant cardiovascular disease burden and mortality, particularly in low/ middle-income countries (LMICs). ${ }^{3}$ It is estimated that approximately 319400 deaths occur annually due to RHD, with an approximated morbidity of 10.5 million disability-adjusted lifeyears. ${ }^{14}$ Pakistan ranks among the top three countries that contribute to the highest estimated numbers of RHD-related deaths at 18900 deaths annually, only to be preceded by India and China. ${ }^{1}$ In Pakistan, the reported prevalence of RHD ranges from as low as 6/1000 population in adults to $22 / 1000$ population in school-going children due to differences in screening protocols used. ${ }^{56}$ However, the true global burden of RHD still remains undetermined due to the lack of sensitive screening 
criteria. ${ }^{7}$ With the updated 2012 WHFcriteria for echocardiographic diagnosis of RHD, there is evidence to support that the RHD burden is higher than previously recorded. ${ }^{7}$

In LMICs, many communities are exposed to recurrent group A streptococcal infections due to increasing urbanisation and overcrowding. ${ }^{3}$ These often untreated infections lead to repeated insults to the cardiac valves, further enhancing valvular dysfunction. Early diagnosis of valvular damage in susceptible populations is fundamental in providing timely prophylaxis and preventing further cardiac morbidity. ${ }^{2}$ Cost-effective analysis of RHD prevention strategies shows that echocardiographic screening and secondary prophylaxis using penicillin is the best strategy to ensure cost-effective care to the individual as well as its impact on the healthcare system. ${ }^{8}$ The incremental cost-effectiveness ratio which compares cost with quality-adjusted life-years was approximately US $\$ 15.39$ for echocardiography and prophylaxis for individuals with RHD as compared with throat swab and antibiotic treatment which would cost US\$266.39. ${ }^{8}$

\section{Traditional screening and diagnosis}

Echocardiography has been proposed as the first-line screening tool for RHD. The prevalence of RHD detected by echocardiogram screening has been shown to be $5-10$ times higher as compared with those by clinical examination. ${ }^{9} 10$ In a recent systematic review by Roberts KV et al, the pooled prevalence of RHD detected by cardiac auscultation was 2.9 per 1000 (95\% CI 1.7 to 5.0) while with echocardiography, this number increased to 12.9 per 1000 people $(95 \%$ CI 0.9 to 18.6$).{ }^{11}$ Despite its accuracy, standard echocardiograms are costly and require specially trained staff, making it an impractical choice to use for mass screening of subclinical RHD. ${ }^{12}$ Recent studies have investigated the use of the handheld echocardiography device, a relatively low-cost machine that can be operated by less skilled healthcare providers, for the first-line screening of subclinical RHD. ${ }^{12}$ The criterion for subclinical RHD screening using handheld echocardiography is MR jet length of $\geq 1.5-2.0 \mathrm{~cm}$ or the presence of AR in the anterior view across several studies. ${ }^{12}$ The sensitivity for borderline and/or definite RHD varied from $73 \%$ to $78.9 \%$ and the specificity ranged between $82.4 \%$ and $87.3 \%$. Further, the sensitivity for definite cases of RHD was approximately $98 \%$ in both studies evaluating handheld echocardiogram criteria. ${ }^{12}{ }^{13}$ Despite the evidence to support handheld echocardiogram screening, no literature is available on its implementation in Pakistan.

\section{Mass screening tool using artificial intelligence}

DASlinked to smartphone-based applications help augment cardiac sounds and provide PCG and ECG waveform data. ${ }^{14}$ PCG, one of the earliest technical approaches for medical signal analysis, is a fundamental method for diagnosing a variety of cardiovascular disorders, such as coronary heart disease, hypertension and arrhythmia. ${ }^{15}$ Although measuring different physical quantities, both an ECG and a PCG are sequential measurements of heart activity used to distinguish normal from abnormal heart function. ${ }^{16}$ Rijuven i2Dtx Cardio Sleeve is a DAS that has achieved a specificity of $94 \%$ and a sensitivity of $91 \%$ in detecting murmurs. ${ }^{14}$ These relatively user-friendly and cost-effective stethoscopes make for an ideal tool in the mass screening of subclinical RHD. Furthermore, machine learning algorithms trained on data from DAS may potentially facilitate in the detection of subclinical RHD without the need for echocardiography, thus identifying the population that may benefit from secondary prophylaxis of RHD.

To achieve the automatic classification of ECG and PCG signals, several approaches have been proposed using classic signal processing techniques, including the Fourier transform, ${ }^{17}$ principle component analysis, ${ }^{18}$ wavelet transform $^{19}$ and the hidden Markov method. ${ }^{20}$ In addition, some machine learning methods, such as artificial neural networks, ${ }^{21}$ support vector machines (SVMs) ${ }^{22}$ least squares $\mathrm{SVMs}^{23}$ and extreme learning machines, ${ }^{24}$ have also been developed for signal classification. However, these methods have some limitations such as requirements for large amount of prior knowledge, manual features selection, and high computational time and complexity. ${ }^{25}$ Recently, convolutional neural networks (CNNs) have proven to be a useful tool in signal and data classification applications. ${ }^{26}$ They are inspired by biological neural networks, thus making CNN-based methods superior in pattern recognition, classification and learning signal structure. In biomedical applications, CNN-based methods have been used for classification in skin cancer, ${ }^{27}$ electromyography signal classification ${ }^{28}$ and ECG classification reference. ${ }^{27}$ Chowdhury et al used a simple long short-term memory (LSTM) network for ECG and PCG classification. ${ }^{27}$ The LSTMs are a type of recurrent neural networks (RNNs) that are capable of learning and detecting long-term dependencies in the input data. ${ }^{25}$ The RNNs are different from the traditional neural networks as they have an internal state that represents context information by storing past input information, thus providing an advantage in classification and prediction tasks. In current advanced technology era, artificial intelligence (AI)-based mass screening tool can act as a bridge to reduce the gap between standard clinical auscultation and echocardiography in resource-limited settings.

Hence, the objectives of this study are (1) to determine the frequency of subclinical RHD in school-going children in Karachi, Pakistan and (2) to train a deep learning algorithm to predict subclinical RHD using auscultatory waveform data from the digital stethoscope.

\section{METHODS}

\section{Study design setting and population}

This will be a cross-sectional study conducted in a group of schools in Karachi (Pakistan). The group runs schools on charity basis and caters to children from low socioeconomic families.

\section{Eligibility criteria}

We will include children between the ages of 5 and 15 years. Any history of diagnosed congenital heart disease 


\section{Box 1 Handheld echocardiography screening protocol}

Parasternal long axis view

1. Clip 2D image; zoom on mitral valve and aortic valve.

2. Place colour box on mitral valve only and pan through valve. Clip to show most presence of MR.

3. Measure the maximum jet length from point of vena contracta to end.

4. Place colour box on aortic valve only and pan through valve. Clip to show most presence of AR.

Apical four-chamber view

1. Clip $2 \mathrm{D}$ image, place focus at valve level.

2. Place colour box on mitral valve and pan through valve. Clip to show most presence of MR.

3. Measure the maximum jet length from point of vena contracta to end.

Apical five-chamber view

1. Clip 2D image; may zoom on left ventricular outflow tract (LVOT) or narrow sector to only visualise LVOT.

2. Place colour box on aortic valve and pan through valve. Clip to show most presence of AR.

(CHD) or CHD detected during echocardiogram performed during the study will be excluded.

\section{Sample size calculation}

According to Watkins et al, the mean standardised prevalence of RHD found in South Asia is $11 / 1000 .{ }^{1}$ Using this prevalence, and assumptions of $90 \%$ statistical power, $5 \%$ level of significance and half of the width of the stated CI as precision, a sample size of 1669 was calculated using the Open Epi sample size calculator. To account for possible dropouts during the study, we will enrol 1700 children.

\section{Research assistants' training}

A workshop will be conducted by investigators to train the research staff on the study questionnaires, obtaining data from the DAS and performing echocardiograms using the HAND criteria.

\section{Recruitment}

The selection of students will be based on random sampling.

The trained research assistants (RAs) will screen children during non-instruction school hours. An estimated 8-10 children will be evaluated per day throughout the 6-day school week. To ensure patient privacy, each student will be screened individually, and only female RAs will be allowed to examine female students. Limited exposure of the precordium will be required, but adequate measures will be taken to ensure patient comfort.

\section{Measurement of outcomes}

Demographic data

This will include age in years, school grade, address, gender, weight in kilogram, height in centimetres, heart rate per minute, systolic and diastolic blood pressure $(\mathrm{mm}$ $\mathrm{Hg}$ ) by sphygmanometer one reading at start of screening and temperature in centigrade $\left({ }^{\circ} \mathrm{C}\right)$.

\section{DAS auscultation protocol}

The RAs will perform the DAS examination consisting of auscultation over four sites: the right (aortic valve area) and left (pulmonary valve area) upper parasternal area at second intercostal space, the apex of the heart (mitral valve) at left fifth intercostal space, mid-clavicular line and left lower parasternal area at fourth intercostal space (tricuspid valve).

The PCG and ECG data obtained from the DAS will be uploaded on a smartphone-based app using a unique study ID.
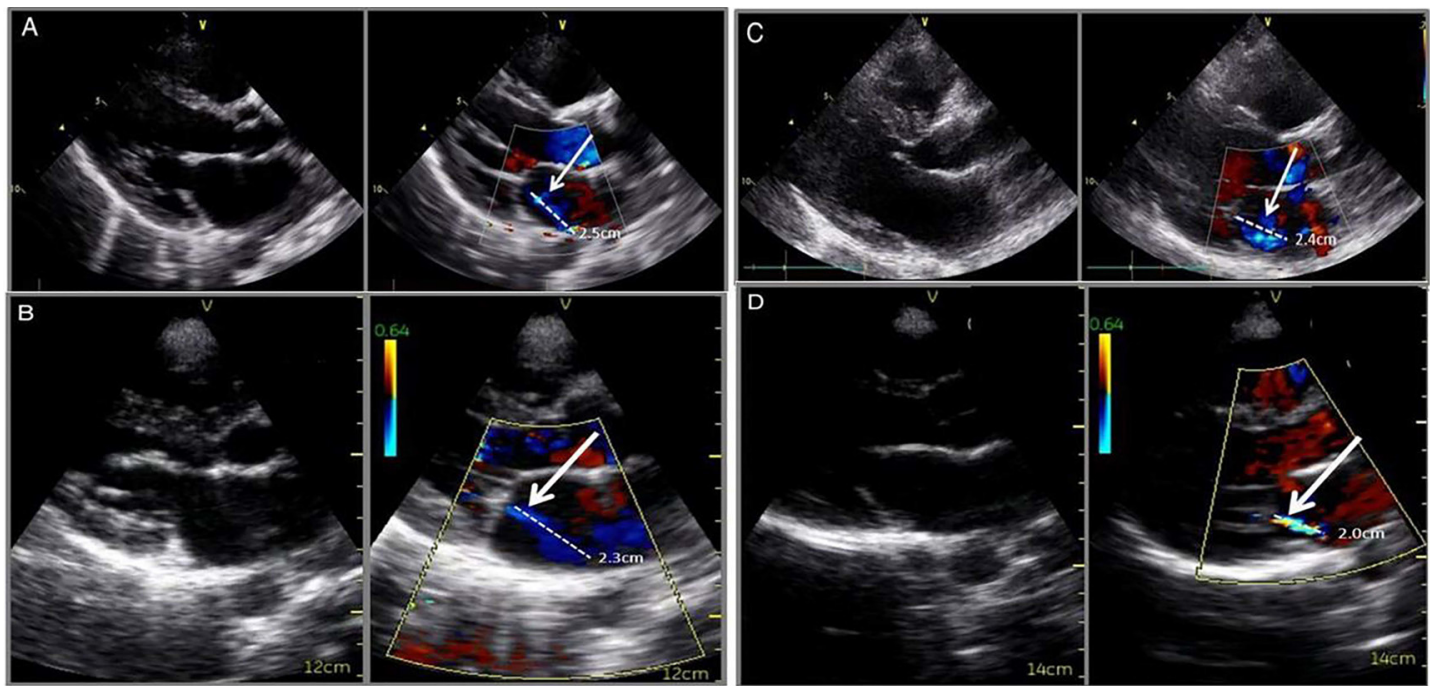

Figure 1 Paired standard/handheld echocardiographic images. With permission to reproduce from Elsevier publisher (https:// doi.org/10.1016/j.echo.2013.09.013). Paired standard/handheld echocardiographic images in patients with rheumatic heart disease (RHD). (A,B) Definite RHD. (C,D) Borderline RHD. White arrows point to the dashed white line showing the length of valvular regurgitation. 


\section{Echocardiography protocol}

Handheld echocardiography

Following auscultation, screening echocardiography will be performed using GE Vscan. Protocol for views and frames is specified in box 1 according to handheld criterion. ${ }^{13}$ Cases with MR jet $\geq 1.5 \mathrm{~cm}$, or the presence of $\mathrm{AR}$ in any view will be labelled as suspected subclinical RHD and eligible for further screening through standard echocardiography.

\section{Standard echocardiography}

The suspected subclinical RHD cases on handheld echocardiography will undergo standard echocardiography by a paediatric cardiologist on Vivid iq machine (GE Healthcare, Chicago Illinois, USA). For echocardiography, machine settings following Nyquist limits for colour Doppler echocardiography will be set on maximum to avoid overestimation of jet length. Images for assessment of valvular and chordal thickness will be acquired with harmonics turned off and probes with variable frequency set on $\geq 2.0 \mathrm{MHz}$. Gain settings will be adjusted to achieve optimal resolution. All other settings (including depth, sector size and focus) will be optimised to achieve maximal frame rate (ideally 30-60 frames per second) and resolution. ${ }^{28}$ Borderline and definite RHD will be decided based on WHF criteria (online supplemental file 1) (figure 1). Protocol for views and frames for standard echocardiogram has been specified in box 2 .

\section{Deep learning protocols}

In this study, we will use the deep neural networks, that is, fully connected LSTMs (FC-LSTMs) and convolution LSTMs (Conv-LSTMs), for the data classification task. These deep learning networks typically require fixed dimension inputs. In case of sequential data, such as PCG, a sequence-to-sequence autoencoder will be used in training and learning stages to create same size inputs. The classification algorithms will be employed on ECG, PCG and on their hybrid version (ECG +PCG). We will use a simple network architecture where a single input neuron connected to the input signal will use several hidden layers of LSTMs. These hidden layers extract the signal features for classification and are connected with the output layer. The squared error of the output layer activation will be used as objective function for the result validation (figure 2 ).

\section{Quality control}

To assess proper conduction and interpretation of echocardiograms and DAS auscultation, a paediatric cardiologist will observe the RAs for the initial days of screening and will check all images weekly.

For suspected subclinical RHD cases which undergo standard echocardiography, two trained paediatric cardiologists will independently review the echocardiography to confirm the diagnosis.

Confirmed cases with subclinical RHD will be referred to a cardiologist for further management. Those children
Box 2 Standard echocardiography protocol

Parasternal long axis view

1. Clip 2D image; zoom on mitral valve and aortic valve.

2. Place colour box on mitral valve only and pan through valve. Clip to show most presence of MR.

3. Through calliper, measure MR jet length from point of vena contracta until end.

4. Through calliper, measure maximum thickness of anterior mitral valve leaflet.

5. Place colour box on aortic valve only and pan through valve. Clip to show most presence of AR.

6. In the presence of AR, perform M-mode through aortic valve with colour on to show presence of AR in systole.

Parasternal short axis view

1. Clip 2D of short axis image at level of mitral valve.

2. Place colour box on mitral valve orifice and clip to show where MR is originating.

\section{Apical four-chamber view}

1. Clip $2 D$ image, place focus at valve level.

2. Turn on tissue velocity imaging (TVI), turn off visibility and clip image-ensure that sector begins at the tip of the sector and includes entire heart.

3. Place colour box on mitral valve and pan through valve. Clip to show most presence of MR.

4. Pulse wave (PW) mitral valve inflow.

5. Continous wave (CW) doppler on mitral valve to include any regurgitation.

6. Tissue Doppler lateral free wall of left ventricle (LV).

7. Tissue Doppler medial septum of LV.

8. Decrease depth to mid-atria and focused on image quality of both the LV and right ventricle (RV)-ensure that frame rate is above 50 or greater or equal to heart rate, clip 2D image.

9. Through calliper, measure MR jet length from point of vena contracta until end.

Apical five-chamber view

1. Clip 2D image; may zoom on left ventricular outflow tract (LVOT) or narrow sector to only visualise LVOT.

2. Place colour box on aortic valve and pan through valve. Clip to show most presence of AR.

3. PW LVOT.

4. CW doppler on aortic valve, take additional clip with doppler baseline lowered in the presence of significant AR to show antegrade regurgitation.

who will need financial assistance will get support from a previously established endowment CHD fund.

The study flow diagram has been detailed in figure 3 .

\section{Data analysis plan}

Data analysis will be performed using SPSS V.20.0. Data will be reported as percentages and means \pm SD where appropriate. The frequency of borderline and definite RHD will be calculated based on true positive (TP) cases diagnosed on standard echocardiography.

The significance of the results will be confirmed using standard hypothesis testing methods, such as analysis of variance (ANOVA) or multivariate ANOVA to compute $p$ value and/or $f$ value. These two values will decide if the null hypothesis should be rejected. In this study, an 


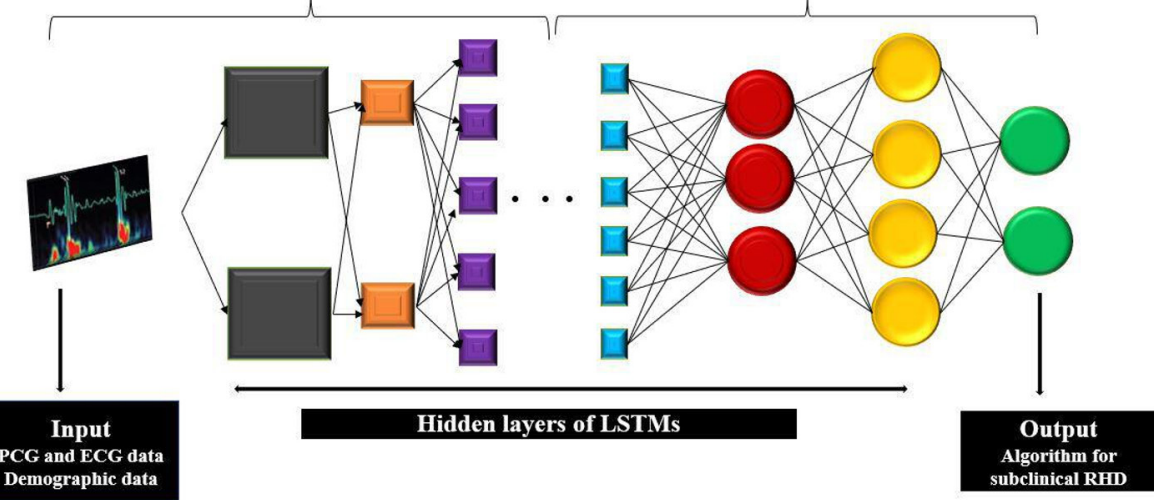

Figure 2 Deep learning schematic diagram. Deep learning model using long short-term memory (LSTM) and its variant, convolution LSTMs and fully connected LSTMs. PCG, phonocardiogram; RHD, rheumatic heart disease.

FC-LSTM or Conv-LSTM is employed as a tool for distinguishing between a normal patient and patient with RHD. The evaluation will be performed with a confusion matrix which predicts the model performance by comparing the

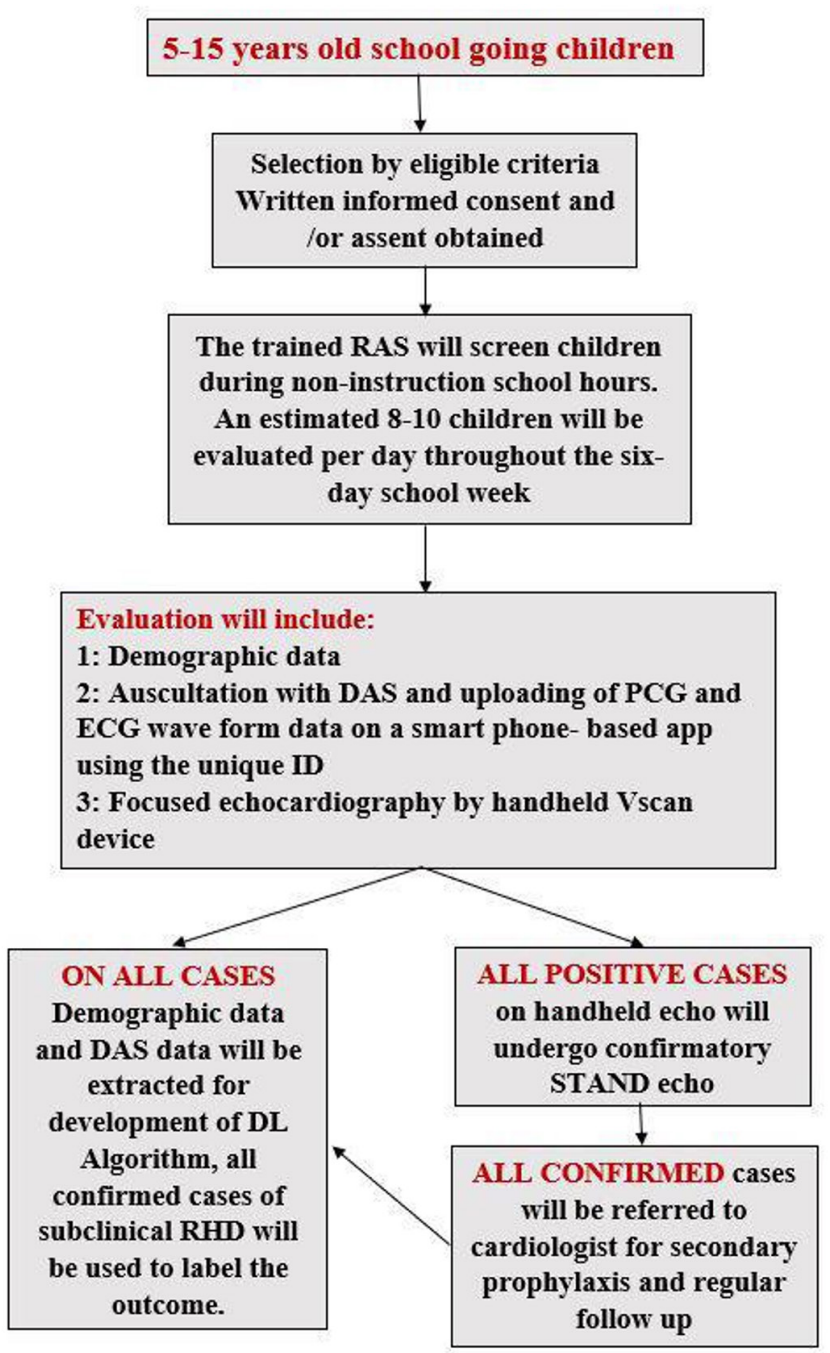

Figure 3 Study flow diagram. DAS, digital auscultatory stethoscope; DL, deep learning; RAs, research assistants; $\mathrm{RHD}$, rheumatic heart disease. model prediction against the true values. The confusion matrix is a $2 \times 2$ matrix, contains $\mathrm{TP}$, true negative $(\mathrm{TN})$, false positive (FP) and false negative $(\mathrm{FN})$ values. The TP and $\mathrm{TN}$ values represent the correctly predicted classifications, while FP and $\mathrm{FN}$ values represent the incorrectly predicted classifications. An example of confusion matrix is given in online supplemental file 2 .

Using the confusion matrix, we can calculate different parameters for evaluation. In our case, the two interesting parameters are the sensitivity and the specificity. The sensitivity, also called the TP rate (TPR), represents how well the classifier predicts the patients with RHD correctly, giving the input ECG and PCG data. The specificity indicates how well the classifier predicts normal patients. With an optimised classifier, the goal is to achieve high sensitivity and specificity. For a given classifier, the sensitivity and specificity are calculated using the following equations:

$$
\begin{aligned}
& \text { Sensitivity }=\frac{T P}{F N+T P} \\
& \text { Specificity }=\frac{T N}{F P+T N}
\end{aligned}
$$

In addition, we will also use the receiver operating characteristic (ROC) curve, which is a graphical plot to analyse a binary classifier performance, and area under the curve (AUC) value for the classifier evaluation. The ROC curve uses the TPR and FP rate (FPR), these rates are then plotted against each other together with a reference line. The AUC value is calculated from the TPR and FPR and is between 0 and 1 which represents the area under the ROC curve. A high AUC (closer to 1) implies a good performing classifier, while a low AUC (below 0.5) indicates worse than randomly guessing.

\section{Potential impact}

From a public health perspective, determining the frequency of subclinical RHD in children is an essential step to estimate the burden of disease at an early stage and initiate the enforcement of secondary prophylaxis. Increased emphasis is required for early detection of subclinical RHD, which can be facilitated through 
handheld devices such as the DAS along with machine learning algorithms to screen young children, without the need for community-based echocardiography. If implemented on a larger scale, these screening tools could revolutionise the detection and treatment of RHD within communities and improve disease outcomes.

\section{Patient and public involvement}

RHD is a fairly common problem at our part of world, majority of patients who have suffered in this lesion belong to poor-resource setting that not only means lack of financial support and disease awareness but access to care as well. All these factors not only contribute in repeated bouts of rheumatic fever leading to significant RHD. Detection of patients who have had an episode of rheumatic fever and have subclinical RHD provides the best window for prevention of advanced RHD by providing prophylaxis against repeated streptococcal infection. Detection of this stage on clinical auscultation is not reliable as cases are missed. Echocardiography is the gold standard but not a feasible solution for screening at population level. Despite adequate counselling in outpatient visit, the financial constraints and lack of access are major hurdles to patient's compliance for follow-up. On basis of these observations, we developed our research proposal to develop an AI algorithm by using waveform data of digital stethoscope to detect subclinical RHD. This will allow for screening in school. Our study population includes school-going children rather than patients with established RHD to detect subclinical RHD. Before enrolment in this study, informed consent or assent will be taken after explaining the purpose of the study and we will also provide the assurance to patients/parents that study finding will be confidential and disseminated to parents/patients directly by the physician and in case of findings of RHD, children will be referred to the cardiologist for further evaluation.

Contributors $\mathrm{FA}, \mathrm{BH}$ and $\mathrm{DC}$ have contributed equally to the study concept, designing and developing the protocol. FA, HA and ZB have contributed to protocol writing. ZH contributed to methodology and statistical writing. MH and SA contributed to machine learning, designing the methods on how waveform data will be processed.

Funding This study was funded by the Aga Khan University, University Research Council grant (URC grant 191003).

Competing interests None declared.

Patient consent for publication Not required.

Provenance and peer review Not commissioned; externally peer reviewed.

Supplemental material This content has been supplied by the author(s). It has not been vetted by BMJ Publishing Group Limited (BMJ) and may not have been peer-reviewed. Any opinions or recommendations discussed are solely those of the author(s) and are not endorsed by BMJ. BMJ disclaims all liability and responsibility arising from any reliance placed on the content. Where the content includes any translated material, BMJ does not warrant the accuracy and reliability of the translations (including but not limited to local regulations, clinical guidelines, terminology, drug names and drug dosages), and is not responsible for any error and/or omissions arising from translation and adaptation or otherwise.

Open access This is an open access article distributed in accordance with the Creative Commons Attribution Non Commercial (CC BY-NC 4.0) license, which permits others to distribute, remix, adapt, build upon this work non-commercially, and license their derivative works on different terms, provided the original work is properly cited, appropriate credit is given, any changes made indicated, and the use is non-commercial. See: http://creativecommons.org/licenses/by-nc/4.0/.

ORCID iDs

Fatima Ali http://orcid.org/0000-0001-8899-7826

Zahra Hoodbhoy http://orcid.org/0000-0002-0439-8293

\section{REFERENCES}

1 Watkins DA, Johnson CO, Colquhoun SM, et al. Global, regional, and national burden of rheumatic heart disease, 1990-2015. N Engl J Med 2017;377:713-22.

2 Sika-Paotonu D, Beaton A, Raghu A, et al. Acute Rheumatic Fever and Rheumatic Heart Disease. In: Ferretti JJ, Stevens DL, Fischetti VA, eds. Streptococcus pyogenes : Basic Biology to Clinical Manifestations. Oklahoma City (OK: University of Oklahoma Health Sciences Center, 2016.

3 Carapetis JR, Steer AC, Mulholland EK, et al. The global burden of group A streptococcal diseases. Lancet Infect Dis 2005;5:685-94.

4 Fever WHOSGoR, Rheumatic Heart D, World Health O. Rheumatic fever and rheumatic heart disease : report of a WHO expert consultation, Geneva, 20 October - 1 November 2001. Geneva World Health Organization. 2004.

5 Rizvi SF, Khan MA, Kundi A, et al. Status of rheumatic heart disease in rural Pakistan. Heart 2004;90:394-9.

6 Remenyi B, Carapetis J, Wyber R, et al. Position statement of the world heart Federation on the prevention and control of rheumatic heart disease. Nat Rev Cardiol 2013;10:284-92.

7 Rothenbühler M, O'Sullivan CJ, Stortecky S, et al. Active surveillance for rheumatic heart disease in endemic regions: a systematic review and meta-analysis of prevalence among children and adolescents. Lancet Glob Health 2014;2:e717-26.

8 Manji RA, Witt J, Tappia PS, et al. Cost-Effectiveness analysis of rheumatic heart disease prevention strategies. Expert Rev Pharmacoecon Outcomes Res 2013;13:715-24.

9 Sadiq M, Islam K, Abid R, et al. Prevalence of rheumatic heart disease in school children of urban Lahore. Heart 2009;95:353-7.

10 Reeves BM, Kado J, Brook M. High prevalence of rheumatic heart disease in Fiji detected by echocardiography screening. $J$ Paediatr Child Health 2011;47:473-8.

11 Roberts KV, Brown ADH, Maguire GP, et al. Utility of auscultatory screening for detecting rheumatic heart disease in high-risk children in Australia's Northern Territory. Med J Aust 2013;199:196-9.

12 Lu JC, Sable C, Ensing GJ, et al. Simplified rheumatic heart disease screening criteria for handheld echocardiography. J Am Soc Echocardiogr 2015;28:463-9.

13 Ploutz M, Lu JC, Scheel J, et al. Handheld echocardiographic screening for rheumatic heart disease by non-experts. Heart 2016;102:35-9.

14 Kocharian A, Sepehri A-A, Janani A, et al. Efficiency, sensitivity and specificity of automated auscultation diagnosis device for detection and discrimination of cardiac murmurs in children. Iran J Pediatr 2013;23:445-50.

15 , Mozaffarian D, Benjamin EJ, Go A, et al, Writing Group Members. Executive Summary: Heart Disease and Stroke Statistics--2016 Update: A Report From the American Heart Association. Circulation 2016;133;): :447-54. vol..

16 Joyanta Kumar Roy Tsr, Subhas Chandra Mukhopadhyay. heart sound: detection and analytical approach towards diseases. Modern Sensing Technologies 2019:103-45.

17 Chauhan S, Wang P, Sing Lim C, et al. A computer-aided MFCCbased HMM system for automatic auscultation. Comput Biol Med 2008;38:221-33.

18 Abdi H, Williams LJ. Principal component analysis. WIREs Comp Stat 2010;2:433-59.

19 Li C, Zheng C, Tai C. Detection of ECG characteristic points using wavelet transforms. IEEE Trans Biomed Eng 1995;42:21-8.

20 Coast DA, Stern RM, Cano GG, et al. An approach to cardiac arrhythmia analysis using hidden Markov models. IEEE Trans Biomed Eng 1990;37:826-36.

21 Yang J, Singh H, Hines EL, et al. Channel selection and classification of electroencephalogram signals: an artificial neural network and genetic algorithm-based approach. Artif Intell Med 2012;55:117-26.

22 Quitadamo FC LR, Sbernini L, et al. Support vector machines to detect physiological patterns for EEG and EMG-based humancomputer interaction: a review. Journal of Neural Engineering 2017;4. 
23 Lima CAM, Coelho ALV, Eisencraft M. Tackling EEG signal classification with least squares support vector machines: a sensitivity analysis study. Comput Biol Med 2010;40:705-14.

24 Ding S, Zhang N, Xu X, et al. Deep extreme learning machine and its application in EEG classification. Mathematical Problems in Engineering 2015;2015:1-11.

25 Bengio Y, Courville A, Vincent P. Representation learning: a review and new perspectives. IEEE Trans Pattern Anal Mach Intell 2013;35:1798-828.

$26 \mathrm{Kim}$ Y. Convolutional neural networks for sentence classification. In Proceedings of the 2014 Conference on Empirical Methods in
Natural Language Processing (EMNLP), Doha, Qatar. Association for Computational Linguistics, 2014:1746-51.

27 Chowdhury MEH, Khandakar A, Alzoubi K, et al. Real-Time SmartDigital stethoscope system for heart diseases monitoring. Sensors 2019;19. doi:10.3390/s19122781. [Epub ahead of print: 20 Jun 2019].

28 Reményi B, Wilson N, Steer A, et al. World heart Federation criteria for echocardiographic diagnosis of rheumatic heart disease-an evidence-based guideline. Nat Rev Cardiol 2012;9:297-309. 